\title{
A Fast Macroblock Mode Decision Algorithm for the Baseline Profile in the H.264 Video Coding Standard
}

\author{
Chang-Hsing Lee, Cheng-Chang Lien, Jau-Ling Shih, and Ping-Yu Lin \\ Department of Computer Science and Information Engineering \\ Chung Hua University, Hsinchu, 300 Taiwan \\ $\{$ chlee,cclien, sjl,m09302036\}@chu.edu.tw
}

\begin{abstract}
A fast macroblock (MB) mode decision algorithm is proposed to reduce the computational complexity of H.264 video coding. First, a SKIP mode detection algorithm is developed to detect as many "skipped" MB as possible. As a result, the encoding functions associated with all inter modes and all intra modes can be omitted. In addition, an early inter mode termination mechanism is developed to disable some inter modes and all intra modes during the motion estimation process. Compared with JM_FFS, the proposed algorithm can reduce $71.35 \%$ of the encoding time with a negligible degradation in video quality and compression ratio.
\end{abstract}

Keywords: H.264, Mode decision, SKIP mode detection.

\section{Introduction}

H.264 is the newest video coding standard, which was developed jointly by the ITU-T Video Coding Experts Group (VCEG) and the ISO-IEC Motion Picture Experts Group (MPEG) [1]. H.264 can achieve significant rate distortion (RD) improvements as compared with previous video coding standards such as MPEG2, MPEG-4, H.263, etc. The key features responsible for the improved coding efficiency include variable block-size motion estimation (ME), multiple reference frames, quarter-pixel motion accuracy, context-adaptive variable length coding (CAVLC), in-loop de-blocking filter, multiple spatial prediction modes for intra prediction, etc. However, the improvement in RD performance is achieved at the expense of increased computational burden [2].

For inter coding of each $16 \times 16$ macroblock (MB), the H.264 encoder employs variable block-size ME to effectively remove temporal correlation between neighboring video frames. H.264 supports four inter coding modes, including $I N T E R 16 \times 16, I N T E R 16 \times 8, I N T E R 8 \times 16$, and $P 8 \times 8$, where a $\mathrm{MB}$ is partitioned into $16 \times 16,16 \times 8,8 \times 16$, or $8 \times 8$ blocks. For the $P 8 \times 8$ mode, each $8 \times 8$ block can be further independently partitioned into $8 \times 8,8 \times 4,4 \times 8$, or $4 \times 4$ blocks.

To exploit the spatial correlation between pixels in the same video frame, H.264 supports two intra coding modes: INTRA4 $\times 4$ and INTRA16 $\times 16$. The

T. Wada, F. Huang, and S. Lin (Eds.): PSIVT 2009, LNCS 5414, pp. $784-795,2009$.

(C) Springer-Verlag Berlin Heidelberg 2009 
INTRA4 $\times 4$ mode allows nine prediction modes for each $4 \times 4$ block whereas the $I N T R A 16 \times 16$ mode allows four prediction modes for each $16 \times 16 \mathrm{MB}$. In addition to the inter coding modes and intra coding modes, the H.264 baseline profile supports the $S K I P$ mode for $\mathrm{P}$ slices to improve the RD performance of video sequences in which neighboring MBs move in identical direction. The $S K I P$ mode implies that no motion information and residual signal have to be encoded. That is, only the mode indicator is actually transmitted for the $S K I P$ mode.

The H.264 reference software 3 incorporates an optional rate-distortion optimization (RDO) mode selection mechanism to select the best coding mode among the set of coding modes. For each coding mode, the RD cost (RDcost) is computed by a distortion model using a Lagrangian coefficient given as 44:

$$
J_{\text {mode }}(M)=D_{\text {mode }}(M)+\lambda_{\text {mode }} R(M),
$$

where $M$ is the coding mode, $M \in C O D I N G \_M O D E S=\{S K I P, I N T E R 16 \times$ $16, I N T E R 16 \times 8, I N T E R 8 \times 16, P 8 \times 8, I N T R A 4 \times 4, I N T R A 16 \times 16\}$, $D_{\text {mode }}(M)$ is the distortion measured as the sum of squared difference (SSD) between the original $\mathrm{MB}$ and the reconstructed $\mathrm{MB}$ associated with the coding mode $M, R_{\text {mode }}(M)$ is the overall bit rate required for the encoding of the motion vector, reference frames, residual signals, etc., $\lambda_{\text {mode }}$ is the Lagrangian parameter for mode decision and is a function related to the quantization parameter $(\mathrm{QP})$ :

$$
\lambda_{\text {mode }}=0.85 \times 2^{(Q P-12) / 3} .
$$

All coding modes will be exhaustively examined and the one with the minimum $R D$ cost is selected as the best coding mode, denoted $M^{*}$ :

$$
M^{*}=\arg \min _{M} J_{\text {mode }}(M), M \in C O D I N G \_M O D E S .
$$

Since each MB is encoded multiple times (one for each coding mode), the computational cost of the RDO mechanism is too high and must be reduced. To reduce the computation time, Yin et al. 5] proposed a fast mode decision algorithm based on the assumption that the error surface versus block-size is probably monotonic. Tu et al. [6] used a merging procedure to determine the encoding block size. A small block-size ME is first performed. An adaptive threshold is then employed to determine whether two neighboring blocks can be merged into a larger block based on the difference between the MVs of these two blocks. A merging and splitting procedure based on the correlation of the MVs of different block-size modes is further employed for fast variable block-size ME [7].

Several studies extracted properties from a MB to predict its possible coding modes and thus omit the other coding modes. The prediction mechanisms can be based on the spatial correlation [8, homogeneous region detection 9, spatial homogeneity and the temporal stationary properties [10], both spatial correlation and temporal correlation [1], 12, etc.

The SKIP mode prediction approach has been employed to reduce the motion search in H.263 video coding [13] 14] in which the ME process will be stopped 
if the sum of absolute difference $(\mathrm{SAD})$ at motion vector $(0,0)$ is less than a pre-defined threshold. This idea has been extended to reduce the computational complexity of H.264 video coding [15 16] 17, 18. The SKIP mode is detected by checking whether all the quantized coefficients are all zeros [15] [16], by comparing the $R D$ cost with a predefined threshold [17, or by comparing the RDcost with an estimated $R D$ cost computed from local sequence statistics 18. The computation time is reduced by avoiding ME and subsequent encoding functions for those MBs being identified as "skipped" MBs.

In this study, a fast MB mode decision algorithm will be proposed to reduce the computational complexity of the H.264 encoding process. In the following section, we will describe the proposed fast MB mode decision algorithm. Simulation results are shown in Section 3 to show the efficiency of the proposed method. Finally, a conclusion is given in Section 4.

\section{Proposed Fast Macroblock Mode Decision Algorithm}

The proposed fast MB mode decision algorithm consists of two stages: early SKIP mode decision and early inter mode termination. First, a $S K I P$ mode detection algorithm is developed to detect as many "skipped" MB as possible compared with the previous approaches. By early identifying a "skipped" MB, the encoding functions related to all inter modes and all intra modes can be omitted. During ME process, the early inter mode termination mechanism tries to disable some inter modes and all intra modes when the coding result of an inter mode is good enough in terms of RDcost.

\subsection{Early SKIP Mode Decision}

The SKIP mode refers to the coding mode where no ME or motion compensation (MC) is performed and no residual signal has to be encoded. Thus, if we can determine at early stage that the best coding mode of the current MB is the SKIP mode, all inter modes and all intra modes can be excluded from the mode decision process. Thus, the encoding functions associated with ME/MC, spatial prediction, and mode decision can be omitted. In general, the SKIP mode dominates among other coding modes, particularly for video sequences with slow or uniform motions (for example, Akiyo, Hall, and Mother\&Daughter), as shown in Table 1. From this table, we can see that for every video sequence the larger the QP values, the more the number of "skipped" MBs. Therefore, early detection of the SKIP mode will save a significant amount of encoding time, particularly for slow motion video sequences or at low bit rates.

In H.264 reference software, when $R D O$ mode selection mechanism is enabled, the $R D$ cost of the $S K I P$ mode is evaluated together with other coding modes. When $R D O$ mode selection mechanism is disabled, the best coding mode of a MB is regarded as the SKIP mode if the following conditions are satisfied: (1) the best MC block size is $16 \times 16$; $(2)$ the reference frame is just the previous one; (3) the MV is the same as its predictive motion vector $(P M V)$; (4) all quantized 
Table 1. Percentage of "skipped" MBs (\%)

\begin{tabular}{ccccc}
\hline Sequence & $\mathrm{QP}=28$ & $\mathrm{QP}=32$ & $\mathrm{QP}=36$ & $\mathrm{QP}=40$ \\
\hline Akiyo & 87.17 & 90.82 & 93.70 & 95.78 \\
Hall & 69.86 & 89.58 & 94.86 & 97.17 \\
Mother\&Daughter & 83.87 & 89.33 & 93.32 & 97.08 \\
Silent & 75.86 & 81.08 & 85.64 & 89.75 \\
News & 80.62 & 85.20 & 88.90 & 92.35 \\
Foreman & 34.45 & 47.96 & 59.92 & 68.86 \\
Coastguard & 14.83 & 29.86 & 50.58 & 70.18 \\
Stefan & 23.54 & 30.28 & 39.65 & 52.18 \\
Table_Tennis & 30.12 & 43.60 & 56.66 & 67.11 \\
Mobile\&Calendar & 4.22 & 7.55 & 14.66 & 30.15 \\
\hline
\end{tabular}

transformation coefficients are all zeros. Let $M B_{o}$ and $M B_{P M V}$ denote respectively the original $\mathrm{MB}$ and the $\mathrm{MB}$ located in the reference frame with $\mathrm{MV}$ being $P M V$. Let $R M B_{P M V}$ denote the residual $\mathrm{MB}$ obtained by taking the difference between $M B_{o}$ and $M B_{P M V}$, that is,

$$
R M B_{P M V}(x, y)=M B_{o}(x, y)-M B_{P M V}(x, y), 0 \leq x, y \leq 15 .
$$

In this study, we presume that $M B_{o}$ is highly probable to be a "skipped" $\mathrm{MB}$ if $R M B_{P M V}$ is an all-zero coefficients $\mathrm{MB}(\mathrm{AZCMB}) . \mathrm{A} \mathrm{MB}$ is referred to as an AZCMB if it is decomposed into a number of non-overlapping $4 \times 4$ blocks and every block is an all-zero coefficients block (AZCB) in which all quantized coefficients become zero. Table 2 shows the probability that the best coding mode is the $S K I P$ mode when $R M B_{P M V}$ is an AZCMB. It can be seen that it is highly probable that a MB will be "skipped" if its residual MB is an AZCMB.

The proposed early $S K I P$ mode decision algorithm tries to disable the other coding modes based on the early identification of an AZCMB. To determine whether a $4 \times 4$ block is an AZCB, a direct way is to perform integer transformation and quantization on this block. To save the computation time, several fast approaches have been proposed to detect AZCB without performing transformation

Table 2. Probability of a MB being encoded as the SKIP mode when it is an AZCMB

\begin{tabular}{ccccc}
\hline Sequence & $\mathrm{QP}=28$ & $\mathrm{QP}=32$ & $\mathrm{QP}=36$ & $\mathrm{QP}=40$ \\
\hline Akiyo & 0.9978 & 0.9963 & 0.9958 & 0.9946 \\
Hall & 0.8559 & 0.9637 & 0.9851 & 0.9950 \\
Mother\&Daughter & 0.9920 & 0.9840 & 0.9808 & 0.9815 \\
Silent & 0.9917 & 0.9873 & 0.9846 & 0.9789 \\
News & 0.9934 & 0.9938 & 0.9921 & 0.9908 \\
Foreman & 0.9323 & 0.9310 & 0.9423 & 0.9371 \\
Coastguard & 0.9134 & 0.9374 & 0.9261 & 0.9351 \\
Stefan & 0.9388 & 0.9600 & 0.9737 & 0.9682 \\
Table_Tennis & 0.8591 & 0.8579 & 0.9140 & 0.9449 \\
Mobile\&Calendar & 0.9257 & 0.9238 & 0.9331 & 0.9352 \\
\hline
\end{tabular}


and quantization. Among these approaches, the most effective one is the enhanced AZCB detection algorithm proposed by $\mathrm{Su}[19$. Let $R B(x, y), 0 \leq x, y \leq 3$, denote the $4 \times 4$ residual block being examined whether it is an AZCB. According to the maximum value within the integer transform kernel functions, the transformation coefficients can be divided into three groups (denoted $G_{0}, G_{1}$, and $G_{2}$ ):

$$
G_{i}=\{(x, y) \mid R B(x, y)=2-(x \% 2)-(y \% 2)=i\}, 0 \leq i \leq 2,
$$

where $\%$ is the modular operator. Specifically, $G_{0}=\{(1,1),(1,3),(3,1),(3$, $3)\}, G_{1}=\{(0,1),(0,3),(1,0),(1,2),(2,1),(2,3),(3,0),(3,2)\}$, and $G_{2}=$ $\{(0,0),(0,2),(2,0),(2,2)\}$. Three different thresholds (denoted $T_{0}, T_{1}$, and $T_{2}$ ) associated with these three groups are obtained as follows:

$$
T_{i}=\frac{2^{15+Q P / 6}-f}{C_{i} \times \mathbf{M}(Q P \% 6, i)}, 0 \leq i \leq 2,
$$

where $C_{i}=2^{2-i}$, QP is the quantization parameter in the range of $[0,51], f$ is a constant and equals $2^{\text {qbits } / 6}$ for inter coded blocks or $2^{\text {qbits } / 3}$ for intra coded blocks (here, qbits $=15+Q P / 6$ ), $\mathbf{M}$ is a $6 \times 3$ matrix given by

$$
\mathbf{M}=\left[\begin{array}{llll}
5243 & 8066 & 13107 \\
4660 & 7490 & 11916 \\
4194 & 6554 & 10082 \\
3647 & 5825 & 9362 \\
3355 & 5243 & 8192 \\
2893 & 4559 & 7282
\end{array}\right] .
$$

From the definition of $T_{i}$, it can be shown that $T_{0}<T_{1}<T_{2}$. As a result, different thresholds for detecting AZCB will serve different frequency components. Let $Q B(x, y)$ denote the quantized coefficient of $R B(x, y)$ and $S$ denote the sum of absolute value of $R B(x, y)$ :

$$
S=\sum_{x=0}^{3} \sum_{y=0}^{3}|R B(x, y)| .
$$

According to the relation between $S$ and $T_{i}$, for identifying AZCB different thresholds will be used for variant frequency component. That is, $Q B(x, y)$ is zero if

$$
(x, y) \in G_{i} \text { and } S<T_{i}^{\prime}, 0 \leq i \leq 2,
$$

where $T_{0}^{\prime}=4 T_{0}-5 S_{\max }, T_{1}^{\prime}=2 T_{1}-2 S_{\max }, T_{2}^{\prime}=T_{2}, S_{\max }$ is defined as follows:

$$
S_{\max }=\max _{i} S_{i}, 0 \leq i \leq 3,
$$

where each $S_{i}(0 \leq i \leq 3)$ is defined as follows:

$$
\begin{aligned}
& S_{0}=|R B(0,0)|+|R B(0,3)|+|R B(3,0)|+|R B(3,3)| . \\
& S_{1}=|R B(0,1)|+|R B(0,2)|+|R B(3,1)|+|R B(3,2)| .
\end{aligned}
$$




$$
\begin{aligned}
& S_{2}=|R B(1,0)|+|R B(1,3)|+|R B(2,0)|+|R B(2,3)| . \\
& S_{3}=|R B(1,1)|+|R B(1,2)|+|R B(2,1)|+|R B(2,2)| .
\end{aligned}
$$

Therefore, if the SAD value, $S$, satisfies the following condition, the examined block is definitely an AZCB:

$$
S<\min \left\{4 T_{0}-5 S_{\max }, 2 T_{1}-2 S_{\max }, T_{2}\right\} .
$$

Based on the above description, the enhanced AZCB detection algorithm is described as follows.

Step 1: If $S<T_{2}$, go to Step 2. Otherwise, proceed with the next block. Step 2: If $S<T_{0}, R B(x, y)$ is an AZCB, proceed with the next block. Otherwise, compute the threshold $T_{\text {new }}=\min \left\{4 T_{0}-5 S_{\max }, 2 T_{1}-2 S_{\max }, T_{2}\right\}$.

Step 3: If $S<T_{n e w}, R B(x, y)$ is an AZCB. Otherwise, proceed with the next block.

Experimental results have shown that Su's enhanced AZCB detection algorithm can detect more AZCBs than other methods [19]. However, a large number of definite AZCBs were not detected by Su's algorithm, as shown in Table 3. Note that Su's enhanced AZCB detection algorithm was developed to omit the integer transformation and quantization operations. In this study, the objective of detecting "skipped" MB is to avoid ME/MC and mode decision operations. In fact, the computation cost required for performing transformation and quantization in H.264 is relative low compared with other encoding functions due to the small transformation block size and the availability of efficient integer implementation 20. From the experimental results of a number of test sequences, it can be found that for an AZCB the SAD value, $S$, tends to be less than $2 T_{2}$. Based on this observation, a minor modification of Su's detection algorithm is developed to improve the AZCB detection rate. For those blocks with $S<2 T_{2}$ and are not "skipped" by Su's detection algorithm, integer transformation and quantization will be performed to check whether they are AZCBs. The comparison of the modified algorithm with Su's algorithm in terms of the detection rate of AZCBs is shown in Table 3. It is quite obvious that almost all AZCBs can be detected by the modified algorithm. However, for those non-AZCBs with $S<2 T_{2}$, the computation time for performing integer transformation and quantization would be an overhead. Table 4 shows the statistics of those non-AZCBs with $S<2 T_{2}$ for different video sequences. It can be seen that the overhead $(1.35 \%-28.76 \%)$ is acceptable. The modified AZCB detection algorithm is described as follows:

Step 1: If $S<T_{2}$, go to Step 2. Otherwise, go to Step 4.

Step 2: If $S<T_{0}, R B(x, y)$ is an AZCB, proceed with the next block. Otherwise, compute the threshold $T_{\text {new }}=\min \left\{4 T_{0}-5 S_{\max }, 2 T_{1}-2 S_{\max }, T_{2}\right\}$.

Step 3: If $S<T_{n e w}, R B(x, y)$ is an AZCB. Otherwise, proceed with the next block.

Step 4: If $S<2 T_{2}$, perform integer transformation and quantization to determine whether $R B(x, y)$ is an AZCB. proceed with the next block. 
Table 3. Detection rate of AZCBs by Su's algorithm and the modified algorithm (\%)

\begin{tabular}{ccccccccc}
\hline Sequence & \multicolumn{3}{c}{ Su's algorithm } & \multicolumn{4}{c}{ Modified algorithm } \\
\hline & $\mathrm{QP}=28$ & $\mathrm{QP}=32$ & $\mathrm{QP}=36$ & $\mathrm{QP}=40$ & $\mathrm{QP}=28$ & $\mathrm{QP}=32$ & $\mathrm{QP}=36$ & $\mathrm{QP}=40$ \\
\hline Akiyo & 75.21 & 78.78 & 81.63 & 84.48 & 99.997 & 99.999 & 100.00 & 100.00 \\
Hall & 37.86 & 65.72 & 66.01 & 72.31 & 99.995 & 99.999 & 100.00 & 100.00 \\
Mother\&Daughter & 65.02 & 65.83 & 72.11 & 83.13 & 99.999 & 100.00 & 100.00 & 100.00 \\
Silent & 18.17 & 25.00 & 36.94 & 59.03 & 99.992 & 99.997 & 100.00 & 100.00 \\
News & 49.76 & 61.56 & 64.41 & 69.83 & 99.991 & 99.998 & 99.999 & 100.00 \\
Foreman & 34.72 & 41.29 & 60.82 & 69.71 & 99.989 & 99.999 & 99.999 & 100.00 \\
Coastguard & 13.94 & 8.98 & 30.47 & 52.22 & 99.924 & 99.990 & 99.997 & 100.00 \\
Stefan & 82.87 & 85.09 & 79.89 & 75.49 & 99.954 & 99.980 & 99.979 & 99.999 \\
Table_Tennis & 52.29 & 48.76 & 57.05 & 68.75 & 99.955 & 99.995 & 99.999 & 99.999 \\
Mobile\&Calendar & 69.96 & 65.19 & 63.61 & 45.93 & 99.939 & 99.949 & 99.938 & 99.990 \\
\hline
\end{tabular}

Table 4. Percentage of non-AZCBs with $S<2 T_{2}(\%)$

\begin{tabular}{ccccc}
\hline Sequence & $\mathrm{QP}=28$ & $\mathrm{QP}=32$ & $\mathrm{QP}=36$ & $\mathrm{QP}=40$ \\
\hline Akiyo & 4.95 & 3.49 & 2.21 & 1.35 \\
Hall & 8.57 & 4.53 & 2.33 & 1.44 \\
Mother\&Daughter & 9.71 & 5.91 & 3.28 & 1.60 \\
Silent & 9.16 & 6.94 & 4.96 & 3.12 \\
News & 6.27 & 4.78 & 3.56 & 2.66 \\
Foreman & 15.61 & 12.20 & 9.02 & 6.10 \\
Coastguard & 27.44 & 28.76 & 20.42 & 12.07 \\
Stefan & 11.41 & 15.80 & 16.48 & 14.81 \\
Table_Tennis & 20.91 & 13.81 & 7.65 & 5.36 \\
Mobile\&Calendar & 10.77 & 15.04 & 16.91 & 17.72 \\
\hline
\end{tabular}

\subsection{Early Inter Mode Termination in ME Process}

If $M B_{O}$ is not skipped, the inter modes are examined successively in the order of mode INTER $16 \times 16, I N T E R 16 \times 8, I N T E R 8 \times 16$, and $P 8 \times 8$. For each inter mode $M, \mathrm{ME}$ is performed to find the corresponding $\mathrm{MV}, \mathbf{m v}_{M}$, which minimizes the $R D$ function, $J_{\text {motion }}\left(M B_{o}, M\right)$. In fact, if the present examined inter mode is good enough, it is unnecessary to perform ME for subsequent inter modes. Let $J_{\text {motion }}^{*}\left(M B_{o}, M\right)$ denote the minimum $R D$ cost obtained in the ME process for inter mode $M$. If $J_{\text {motion }}^{*}\left(M B_{o}, M\right)$ is less than a pre-calculated threshold, $T H(M)$, it is presumed that the current coding mode is good enough and thus the other coding modes can be omitted. The major problem is how to determine the threshold. Since there exits high correlation between neighboring video frames, the threshold can be derived by the $R D$ cost of the MBs in the reference frame. Let $S(M)$ denote in the reference frame the set of MBs whose best coding mode is mode $M$, and $\bar{J}_{\text {motion }}(M)$ denote the average $R D$ cost of these MBs, that is,

$$
\bar{J}_{\text {motion }}(M)=\frac{1}{|S(M)|} \sum_{B \in S(M)} J_{\text {motion }}^{*}\left(B, M^{*}(B)\right),
$$


where $|\bullet|$ is the cardinality of a set, $M^{*}(B)$ is the best coding mode of a MB $B$. Similarly, let $S(I N T E R)$ denote the set of inter coded MBs in the reference frame, that is,

$S(I N T E R)=S(I N T E R 16 \times 16) \cup S(I N T E R 16 \times 8) \cup(I N T E R 8 \times 16) \cup S(P 8 \times 8)$.

The average $R D$ cost of these inter coded MBs, $\bar{J}_{\text {motion }}(I N T E R)$, is then defined as follows:

$$
\bar{J}_{\text {motion }}(I N T E R)=\frac{1}{|S(I N T E R)|} \sum_{B \in S(I N T E R)} J_{\text {motion }}^{*}\left(B, M^{*}(B)\right) .
$$

For each mode $M$, the adaptive threshold, $T H(M)$, is then defined as:

$$
T H(M)=\min \left\{\bar{J}_{\text {motion }}(M), \bar{J}_{\text {motion }}(I N T E R)\right\} .
$$

If $J_{\text {motion }}^{*}\left(M B_{o}, M\right)<T H(M)$, the other inter modes as well as all intra modes will be disabled. Note that this early inter mode termination approach is performed during the ME process. As a result, the reduction of computation time is achieved by avoiding unnecessary $\mathrm{ME}(\mathrm{s})$, spatial predictions, and mode decisions of those disabled coding modes. In addition to the threshold-based mode termination approach, an additional early inter mode method based on the assumption that the error surface versus block-size is probably monotonic is developed before examining the time intensive $P 8 \times 8$ mode. Since MEs on block sizes of $16 \times 16$, $16 \times 8$, and $8 \times 16$ have been examined before performing $\mathrm{ME}$ on each $8 \times 8$ block, if $I N T E R 16 \times 16$ achieves the minimum $R D$ cost, the $P 8 \times 8$ mode can be omitted. That is, $P 8 \times 8$ will be disabled if

$$
J_{\text {motion }}^{*}\left(M B_{o}, I N T E R 16 \times 16\right)<J_{\text {motion }}^{*}\left(M B_{o}, I N T E R 16 \times 8\right)
$$

and

$$
J_{\text {motion }}^{*}\left(M B_{o}, I N T E R 16 \times 16\right)<J_{\text {motion }}^{*}\left(M B_{o}, I N T E R 8 \times 16\right)
$$

During mode decision process, the best encoding mode is determined among the SKIP mode, enabled inter modes, and two spatially predictive intra modes, $I N T R A 16 \times 16$ and INTRA4 $\times 4$ if they are enabled. The selection of the best encoding mode is measured in terms of $R D$ cost defined in Eq. (1). The detailed steps of the proposed algorithm are given as follows.

Step 1: Let $M B_{o}$ denote the current MB being encoded. If $M B_{o}$ is an AZCMB, set the best coding mode of $M B_{o}$ the $S K I P$ mode and go to Step 8 .

Step 2: Perform ME for the $I N T E R 16 \times 16$ mode and get the corresponding minimum $R D$ cost, $J_{\text {motion }}^{*}\left(M B_{o}, I N T E R 16 \times 16\right)$. If $J_{\text {motion }}^{*}\left(M B_{o}, I N T E R 16 \times\right.$ 16) $<T H(I N T E R 16 \times 16)$, disable $I N T E R 16 \times 8, I N T E R 8 \times 16, P 8 \times 8$, $I N T R A 16 \times 16$, and INTRA4 $\times 4$, go to Step 7 .

Step 3: Perform ME for the INTER $16 \times 8$ mode and get the corresponding minimum $R D$ cost, $J_{\text {motion }}^{*}\left(M B_{o}, I N T E R 16 \times 8\right)$. If $J_{\text {motion }}^{*}\left(M B_{o}, I N T E R 16 \times\right.$ $8)<T H(I N T E R 16 \times 8)$, disable $I N T E R 8 \times 16, P 8 \times 8, I N T R A 16 \times 16$, and INTRA4 × 4, go to Step 7 . 
Step 4: Perform ME for the INTER $\times 16$ mode and get the corresponding minimum $R D$ cost, $J_{\text {motion }}^{*}\left(M B_{o}, I N T E R 8 \times 16\right)$. If $J_{\text {motion }}^{*}\left(M B_{o}, I N T E R 8 \times\right.$ 16) $<T H(I N T E R 8 \times 16)$, disable $P 8 \times 8, I N T R A 16 \times 16$, and $I N T R A 4 \times 4$, go to Step 7 .

Step 5: If $J_{\text {motion }}^{*}\left(M B_{o}, I N T E R 16 \times 16\right)<J_{\text {motion }}^{*}\left(M B_{o}, I N T E R 16 \times 8\right)$ and $J_{\text {motion }}^{*}\left(M B_{o}, I N T E R 16 \times 16\right)<J_{\text {motion }}^{*}\left(M B_{o}, I N T E R 8 \times 16\right)$, disable $P 8 \times 8$ and go to Step 7.

Step 6: Perform ME for the $P 8 \times 8$ mode.

Step 7: In the mode decision process, calculate the RDcosts of the $S K I P$ mode, all enabled inter modes, and all enabled intra modes. Determine the best coding mode, $M^{*}$, which achieves the minimum RDcost among the SKIP mode and all enabled modes.

Step 8: Proceed with next MB, go to Step 1.

\section{Experimental Results}

The experiments were implemented by using the H.264 reference software JM10.1 provided by the Joint Video Team (JVT). We compared the proposed fast mode decision algorithm with the fast algorithms proposed by Yang et al. [17 and Kannangara et al. 18. The fast full search (JM_FFS) algorithm was tested for comparison. We have tested ten video sequences with different motion activities, including Akiyo, Hall, Mother\&Daughter, Silent, News, Foreman, Coastguard, Stefan, Table_Tennis, and Mobile\&Calendar. Each of them has 100 frames of the CIF format $(352 \times 288)$. The length of a GOP is 10 and all frames within a GOP except the first frame (encoded as I frame) are encoded as $\mathrm{P}$ frames. The motion search range is 16 and the number of reference frame is 1 . RDO and CAVLC are enabled in our experiments. The main encoding parameters are listed in Table 5.

To examine the performance at different bit rates, four QP values, 28, 32, 36, 40 , are tested in our experiments. The comparison of average speed-up factors compared with JM_FFS algorithm is shown in Table 6. It can be seen that the proposed method outperforms Yang's and Kannangara's methods and the average speed-up factors is $71.35 \%$. Table 7 shows the average number of mode searches during ME. This table also indicates that the proposed method requires

Table 5. Encoding parameters for JM-10.1 reference software

\begin{tabular}{cc}
\hline Configuration & Parameters \\
\hline Length of video frames for the simulation & 100 \\
Number of reference frames & 1 \\
Search range for the motion estimation & 16 \\
Hadamard transform for encoding DC components & ON \\
Rate-distortion optimization & ON \\
CAVLC entropy coding & ON \\
Length of GOP & 10 \\
Number of test video sequences (CIF format) & 10 \\
\hline
\end{tabular}


Table 6. Comparison of computation time reduction compared with JM_FFS (S1: our SKIP mode decision method; S2: our early inter mode termination method)

\begin{tabular}{ccccc}
\hline Sequence & \multicolumn{2}{c}{ Yang Kannangara } & S1 & S1+S2 \\
\hline Akiyo & 38.30 & 62.37 & 69.31 & 83.46 \\
Hall & 33.29 & 47.97 & 63.26 & 81.03 \\
Mother\&Daughter & 34.82 & 52.02 & 59.95 & 79.16 \\
Silent & 23.73 & 49.07 & 57.45 & 77.84 \\
News & 31.11 & 55.13 & 63.16 & 80.97 \\
Foreman & 21.43 & 32.43 & 34.31 & 67.17 \\
Coastguard & 8.60 & 15.91 & 18.43 & 61.54 \\
Stefan & 11.82 & 19.03 & 20.38 & 60.66 \\
Table_Tennis & 18.06 & 28.94 & 32.57 & 66.90 \\
Mobile\&Calendar & 3.53 & 4.38 & 7.20 & 54.79 \\
\hline Average & 22.47 & 36.73 & 42.60 & 71.35 \\
\hline
\end{tabular}

Table 7. Comparison of the average number of mode operations

\begin{tabular}{ccccc}
\hline Mode & \multicolumn{2}{c}{ Yang Kannangara } & S1 & S1+S2 \\
\hline$S K I P$ & 35640 & 35640 & 35640 & 35640 \\
$I N T E R 16 \times 16$ & 24682 & 20349 & 17956 & 18007 \\
$I N T E R 16 \times 8$ & 24682 & 20349 & 17956 & 8754 \\
$I N T E R 8 \times 16$ & 24682 & 20349 & 17956 & 5293 \\
$P 8 \times 8$ & 24682 & 20349 & 17956 & 2088 \\
$I N T R A 16 \times 16$ & 24682 & 20349 & 17956 & 1547 \\
$I N T R A 4 \times 4$ & 24682 & 20349 & 17956 & 1547 \\
\hline
\end{tabular}

Table 8. Average objective performance gains (avsnr)

\begin{tabular}{|c|c|c|c|c|c|c|c|c|}
\hline \multirow[t]{2}{*}{ Sequence } & \multicolumn{2}{|c|}{ Yang } & \multicolumn{2}{|c|}{ Kannangara } & \multicolumn{2}{|c|}{ S1 } & \multicolumn{2}{|c|}{$\mathrm{S} 1+\mathrm{S} 2$} \\
\hline & $\begin{array}{l}\text { PSNR } \\
\end{array}$ & Rate(\%) & $\overline{\mathrm{PSNR}}$ & Rate(\%) & PSNR & Rate(\%) & $\begin{array}{l}\text { PSNR } \\
\end{array}$ & iate(\%) \\
\hline Akiyo & -0.34 & 7.33 & 0.01 & -0.22 & -0.01 & 0.14 & -0.06 & 1.22 \\
\hline Hall & -0.02 & 0.53 & 0.02 & -0.44 & 0.05 & -1.15 & -0.07 & 1.37 \\
\hline Mother\&Daughter & -0.73 & 21.54 & 0.01 & -0.03 & -0.01 & 0.41 & -0.08 & 2.05 \\
\hline Silent & -0.22 & 5.49 & 0.00 & -0.02 & -0.01 & 0.30 & -0.14 & 3.56 \\
\hline News & -0.29 & 5.30 & 0.00 & -0.05 & -0.02 & 0.37 & -0.18 & 3.16 \\
\hline Foreman & -0.71 & 20.33 & -0.05 & 1.21 & -0.07 & 1.82 & -0.25 & 6.44 \\
\hline Coastguard & -0.11 & 3.26 & -0.02 & 0.64 & -0.06 & 1.68 & -0.19 & 5.70 \\
\hline Stefan & -0.12 & 2.38 & -0.01 & 0.18 & -0.03 & 0.56 & -0.17 & 3.46 \\
\hline Table_Tennis & -0.37 & 11.14 & -0.05 & 1.31 & -0.07 & 2.07 & -0.24 & 7.19 \\
\hline Mobile\&Calendar & -0.08 & 1.50 & 0.00 & 0.06 & -0.05 & 1.08 & -0.23 & 4.38 \\
\hline Average & -0.30 & 7.88 & -0.01 & 0.27 & -0.03 & 0.73 & -0.16 & 3.85 \\
\hline
\end{tabular}

less number of mode operations than the other two methods. Table 8 gives the average rate and PSNR differences between the RD curves for each fast method and JM_FFS as calculated using the Bjontegaard measurement method 21. From Table 8, we can see that Kannangara's approach yields the best RD 
performance but the reduction in computation time of our proposed approach is much better than Kannangara's approach (71.35\% vs. $36.73 \%$ ), as shown in Table 6 .

\section{Conclusion}

A fast MB mode decision algorithm is proposed to reduce the computation time of H.264 video coding. Before ME, those MBs that are likely to be "skipped" are first identified. Computational saving is achieved without performing variable block-size ME and mode decisions. Further, an early inter mode termination approach is employed to disable some inter modes and all intra modes if the coding result of the current examined inter mode is good enough in terms of RD measure. Compared with JM_FFS, the proposed algorithm can reduce $71.35 \%$ of the encoding time with a negligible degradation in video quality and compression ratio.

\section{Acknowledgements}

This research was supported in part by the National Science Council of R.O.C. under contract NSC-96-2221-E-216-043.

\section{References}

[1] ITU-T: Recommendation H.264: Advanced Video Coding for Generic Audiovisual Services (2003)

[2] Ostermann, J., Bormans, J., List, P., Marpe, D., Narroschke, M., Pereira, F., Stockhammer, T., Wede, T.: Video coding with H.264/AVC: tools, performance and complexity. IEEE Circuits and Systems Magazine 4, 7-28 (2004)

[3] Joint Video Team (JVT) Reference software JM-10.1, http://iphome.hhi.de/suehring/tml/

[4] Sullivan, G.J., Wiegand, T.: Rate-distortion optimization for video compression. IEEE Signal Processing Magazine 15, 74-90 (1998)

[5] Yin, P., Tourapis, H.Y.C., Tourapis, A.M., Boyce, J.: Fast mode decision and motion estimation for JVT/H.264. In: Int. Conf. on Image Processing, vol. 3, pp. 853-856 (2003)

[6] Tu, Y.K., Yang, J.F., Sun, M.T., Tsai, Y.T.: Fast variable-size block motion estimation for efficient H. 264/AVC encoding. Signal Processing: Image Communication 20, 595-623 (2005)

[7] Zhou, Z., Xin, J., Sun, M.T.: Fast motion estimation and inter-mode decision for H. 264/MPEG-4 AVC encoding. Journal of Visual Communication and Image Representation 17, 243-263 (2006)

[8] Li, G.L., Chen, M.J., Li, H.J., Hsu, C.T.: Efficient search and mode prediction algorithms for motion estimation in H.264/AVC. In: IEEE Int. Symp. on Circuits and Systems, vol. 6, pp. 5481-5484 (2005)

[9] Jing, X., Chau, L.P.: Fast approach for H. 264 inter mode decision. Electronics Letters 40, 1050-1051 (2004) 
[10] Wu, D., Pan, F., Lim, K.P., Wu, S., Li, Z.G., Lin, X., Rahardja, S., Ko, C.C.: Fast intermode decision in H. 264/AVC video coding. IEEE Trans. on Circuits and Systems for Video Technology 15, 953-958 (2005)

[11] Yin, M., Wang, H.Y.: An improvement fast INTER mode selection for H.264 joint with spatio-temporal correlation. In: Int. Conf. on Wireless Communications, Networking and Mobile Computing, pp. 1237-1240 (2005)

[12] Khan, N.A., Masud, S., Ahmad, A.: A variable block size motion estimation algorithm for real-time H. 264 video encoding. Signal Processing: Image Communication 21, 306-315 (2006)

[13] Yang, J.F., Chang, S.C., Chen, C.Y.: Computation reduction for motion search in low rate video coders. IEEE Trans. on Circuits and Systems for Video Technology 12, 948-951 (2002)

[14] Zhao, Y., Richardson, I.: Macroblock classification for video encoder complexity management. In: Int. Picture Coding Symposium, pp. 371-376 (2003)

[15] Lee, J., Jeon, B.: Fast mode decision for H.264. In: IEEE Int. Conf.on Multimedia and Expo., pp. 1131-1134 (2004)

[16] Kim, Y., Choe, Y., Choi, Y.: Fast mode decision algorithm for H.264 using AZCB prediction. In: IEEE Int. Conf. on Consumer Electronics, pp. 33-34 (2006)

[17] Yang, L., Yu, K., Li, J., Li, S.: An effective variable block-size early termination algorithm for H. IEEE Trans. on Circuits and Systems for Video Technology 15, 784-788 (2005)

[18] Kannangara, C.S., Richardson, I.E.G., Bystrom, M., Solera, J.R., Zhao, Y., MacLennan, A., Cooney, R.: Low-complexity skip prediction for H. 264 through Lagrangian cost estimation. IEEE Trans. on Circuits and Systems for Video Technology 16, 202-208 (2006)

[19] Su, C.Y.: An enhanced detection algorithm for all-zero blocks in H. 264 video coding. IEEE Trans. on Consumer Electronics 52, 598-605 (2006)

[20] Malvar, H.S., Hallapuro, A., Karczewicz, M., Kerofsky, L.: Low-complexity transform and quantization in H. 264/AVC. IEEE Trans. on Circuits and Systems for Video Technology 13, 598-603 (2003)

[21] Bjontegaard, G.: Calculation of average PSNR differences between RD-curves. In: 13th VCEG-M33 Meeting (2001) 